\title{
Comparative and combined effectiveness of innovative therapies in cancer: a literature review
}

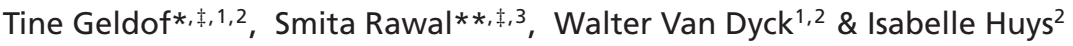 \\ ${ }^{1}$ Healthcare Management Centre, Vlerick Business School, Reep 1, Ghent 9000, Belgium \\ ${ }^{2}$ Pharmaceutical Care \& Pharmaco-economics, KU Leuven, O\&N II, Leuven 3001, Belgium \\ ${ }^{3}$ Department of Pharmaceutical Health Services, Outcomes \& Policy, College of Pharmacy, University of Georgia, Athens 30602, \\ GA, USA \\ *Author for correspondence: Tel.: +32 921097 07; tine.geldof@vlerick.com \\ **Author for correspondence: Tel.: +1 706542 7230; sr54982@uga.edu \\ $¥$ Authors contributed equally
}

To achieve therapeutic innovation in oncology, already expensive novel medicines are often concomitantly combined to potentially enhance effectiveness. While this aggravates the pricing problem, comparing effectiveness of novel yet expensive (concomitant) treatments is much needed for healthcare decisionmaking to deliver effective but affordable treatments. This study reviewed published clinical trials and real-world studies of targeted and immune therapies. In total, 48 studies compared and/or combined multiple novel products on breast, colorectal, lung and melanoma cancers. To a great extent, products evaluated in each study were owned by one manufacturer. However, cross-manufacturer assessments are also needed. Next to costs and intensive market competition, the absence of a regulatory framework enforcing real-world multiproduct studies prevents these from being conducted. Trusted third parties could facilitate such real-world studies, for which appropriate and efficient data access is needed.

First draft submitted: 22 November 2018; Accepted for publication: 13 December 2018; Published online: 8 January 2019

Keywords: clinical studies • combination studies • comparative studies • immune-oncology • literature review • precision-oncology • real-world evidence

\section{Background}

In the past decade, emerging knowledge on the complexity and behavior of cancer cells has resulted in large investments in clinical research, fostering a new era of novel and expensive medicines, which include targeted and immune therapies [1,2]. These medicines shift the 'one-size-fits-all' approach of conventional cytostatics to a targeted cancer- and immune-cell approach, which therefore classifies them as innovative medicines [3]. Today, many novel medicines are entering clinical trials, quickly increasing the number of available, but often unaffordable, treatment options in oncology $[1,2]$. With this evolution, the relative efficacy of these products has become an important issue for healthcare decision-making.

However, while developing innovative therapies is difficult, achieving therapeutic innovation, that is, providing favorable and clinically significant benefits, preferably in an area of unmet need, is even more complicated [3]. Accordingly, the need to concomitantly combine various drugs with complementary mechanisms of action has become apparent, which aggravates the affordability problem [4,5]. For example, significant benefits for HER2overexpressing metastatic breast cancer patients have been demonstrated using concomitant pertuzumab and trastuzumab, two recombinant humanized monoclonal antibodies, as compared with using trastuzumab alone. The combination represented a substantial improvement of 6.3 and 16.3 months in progression-free survival (PFS) and overall survival (OS), respectively [6], however, it comes with a cost of $\$ 713,219$ per quality-adjusted life-years gained, and is under no circumstance likely to be cost-effective [7].

Consequently, comparisons are also highly necessary for concomitant treatments, because nonadditive differences in the costs and benefits mean that separate regulatory decisions are needed [8]. This is important for both 
noncompeting and (in)directly competing products, the latter being defined as those medicines that target the same therapeutic indication and patient (sub)population with similar or dissimilar active substances from different manufacturers.

Unfortunately, the comparative and/or combined effects of these novel treatments are often unassessed, creating considerable concerns regarding the exorbitant and unbalanced nature of novel cancer therapy prices $[9,10]$. This causes regulatory agencies to demand for additional randomized controlled trials (RCTs). However, in turn, clinical trials performing head-to-head comparisons and/or assessing plausible concomitant treatments are highly timeconsuming and expensive [11], with the resulting treatment pathways driving the cost of cancer care ever higher [12]. This is especially true for novel cancer therapies and even more so for forthcoming gene therapies. Presumably, such hurdles withhold manufacturers from conducting multiproduct assessments of novel products in the competitive pharmaceutical environment, especially if they have already obtained market access [11,12].

The objective of this review is to examine those clinical studies that have investigated the efficacy and effectiveness of (competing) innovative therapies in comparison and/or in combination with each other to gain better understanding of the phenomenon. Innovative therapies are defined here as products that enable a personalized treatment approach, such as targeted and immune therapies. First, the methodology used to perform the literature review is described. Subsequently, its results are described for both pre- and postmarket investigations and analyzed in terms of the included products' marketing authorization holders. Following this, the implications of these studies are discussed, together with the importance of real-world investigations for facilitating such assessments.

\section{Methods}

\section{Search strategy}

Two independent authors (TG and SR) conducted a comprehensive search to identify relevant studies in PubMed, EMBASE and the Cochrane Central Register of Controlled Trials (CENTRAL) library from 1 May 2018 to 31 September 2018. Additionally, the websites of the European Society of Medical Oncology, the American Society of Clinical Oncology, the Belgian Society of Medical Oncology, the European Medicines Agency and the clinicaltrials.gov database were searched for ongoing research on innovative anticancer combination therapies. The following keywords, synonyms and MeSH terms were used: 'Combined Modality Therapy', 'Neoplasms', 'Clinical Trials, Phase III as Topic', 'Clinical Trial, Phase IV' and 'Observational Study'. After removing duplicates, we screened all eligible studies in a step-wise manner based on title, abstract and reading the full text. Additionally, we reviewed articles' reference lists, checked clinical trials registers and hand-searched relevant journal proceedings. Only English-language publications were included.

\section{Study designs included in the review}

All study designs, including RCTs of Phase III or IV, open-label studies, observational studies, literature reviews and case reports, that compared or combined innovative anticancer drugs (including targeted and immunologic therapies) were eligible. Efficacy and effectiveness end points assessed in these studies include OS, PFS, response rate and toxicity. We chose to include only breast cancer, colorectal cancer (CRC), non-small-cell lung cancer (NSCLC) and melanoma in our study, these being the four most frequently occurring cancers in Europe.

\section{Analysis}

Two authors (TG and SR) independently searched the literature and clinical trial registers. If there were disputes, the researchers discussed them until they reached consensus. Eligible publications were sorted by product and indication and classified into two groups and four subgroups:

- Premarket studies (RCTs) of innovative cancer treatments

- Comparisons between competitive (potentially combined) products for example pertuzumab versus trastuzumab in breast cancer;

- Combinations of competitive products, for example pertuzumab plus trastuzumab.

- Postmarket (observational) studies of (combined) innovative cancer treatments

- Comparisons between competitive (potentially combined) products;

- Combinations of competitive products.

Studies were combined within these four subgroups when the same medicinal products (i.e., active substances) were assessed within these studies. In addition, a nonexhaustive overview of ongoing studies was constructed. 


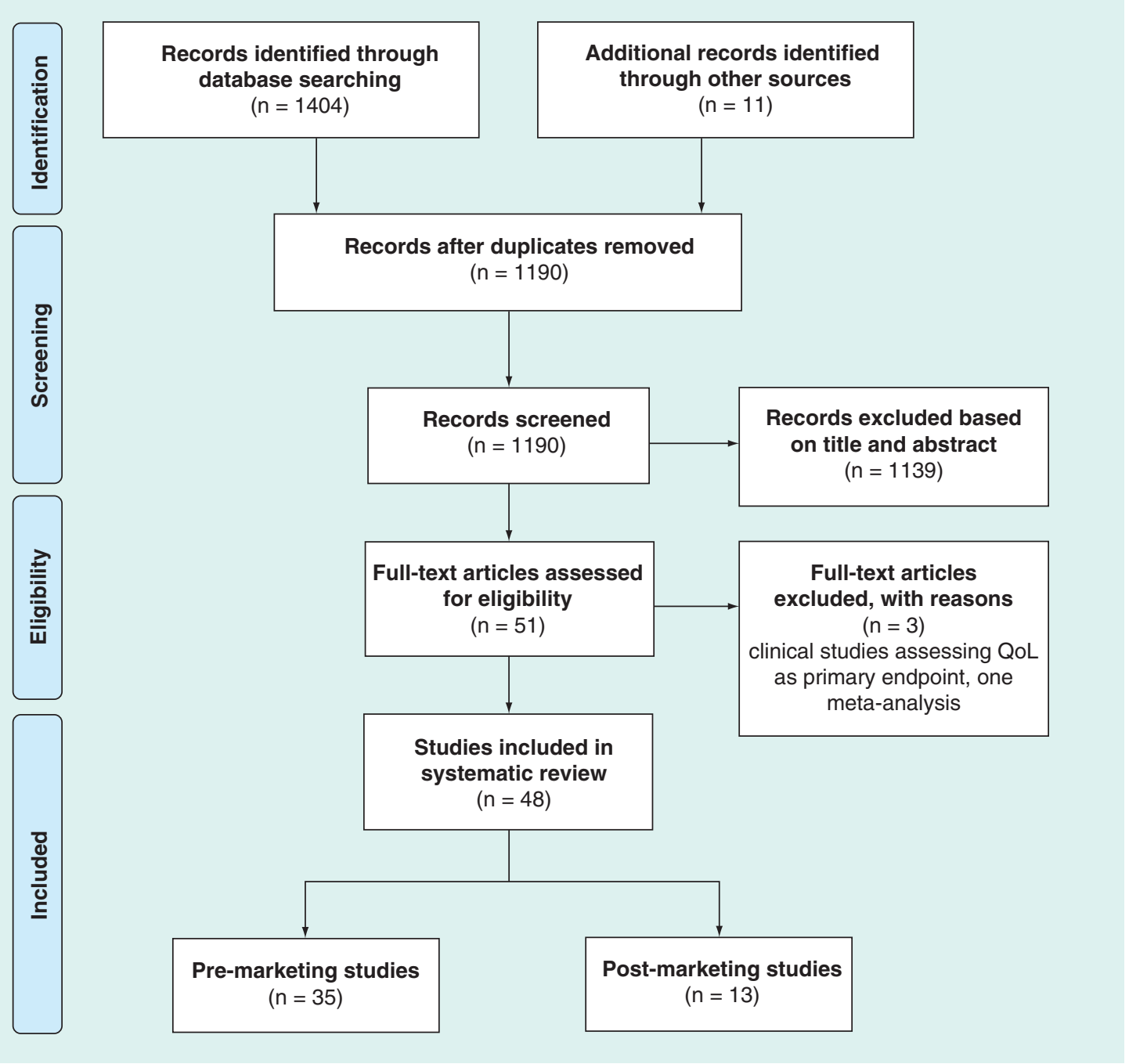

Figure 1. PRISMA flow diagram for reporting a literature review (adapted from the PRISMA statement). QoL: Quality of life.

\section{Results}

In total, 1404 articles were found through the search strategy. After duplicate removal and application of the inclusion and exclusion criteria, 48 relevant articles reporting comparative and combined effectiveness studies of innovative cancer treatments were retained (Figure 1). Of these, 35 and 13 were pre- and post-marketing studies, respectively. A detailed overview of these studies, including summarized information on study designs and conclusions, is presented in the Appendix. After grouping those assessing the same compared and/or combined treatments per indication and combining studies per product assessed, 34 unique comparisons and combinations were found (28 premarket and seven postmarket; Table 1). Below is given a description of these studies for four cancer indications: breast, colorectal, non-small-cell lung and melanoma. In addition, a nonexhaustive overview of 57 ongoing studies is presented in the Appendix.

\section{Breast cancer}

Despite recent therapeutic advances, breast cancer remains the second leading cause of cancer-related death among women [13]. HER2 overexpression in breast cancer is associated with better response to anti-HER2 therapies, such as trastuzumab, in combination with chemotherapy [14]. The pre- and post-marketing comparative and/or combination studies on innovative medicines for breast cancer are described below. 


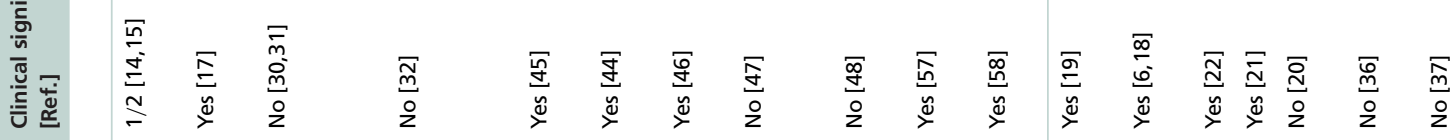

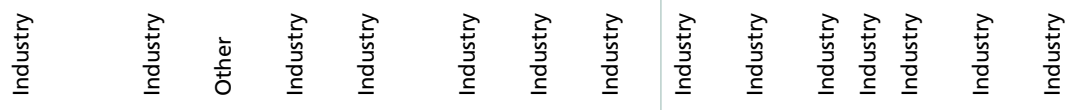

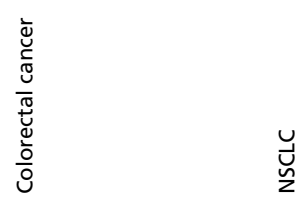<smiles>CCCC</smiles><smiles>C1CCCCC1</smiles>

$\sum_{0}^{x} \sum_{0}^{x} \sum_{0}^{x}$ $\stackrel{\times}{x}$ $\sum_{0}^{x} \sum_{0}^{x}$ $\sum_{0}^{x}$ $\sum_{a}^{x} \underline{x} \sum_{a}^{x} \sum_{a}^{x} \sum_{a}^{x} \sum_{a}^{x} \sum_{a}^{x} \sum_{a}^{x} \sum_{a}^{x} \sum_{a}^{x}$

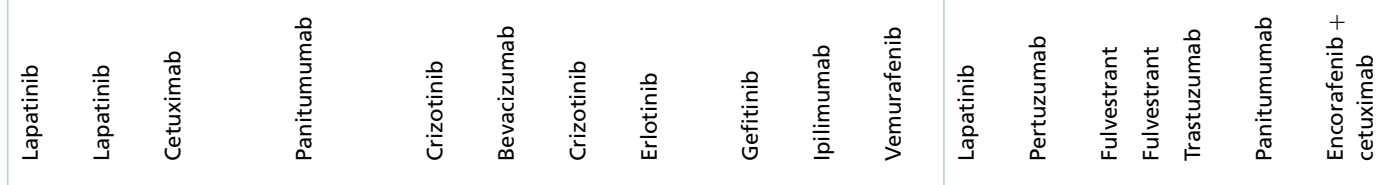

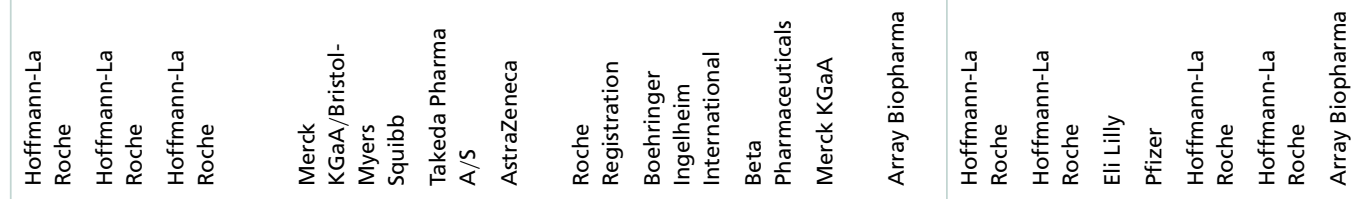

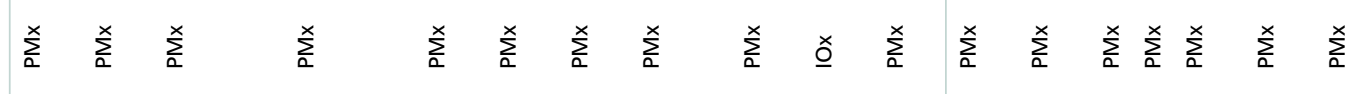

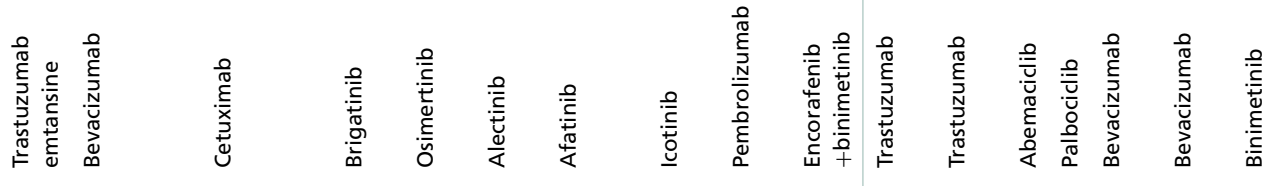




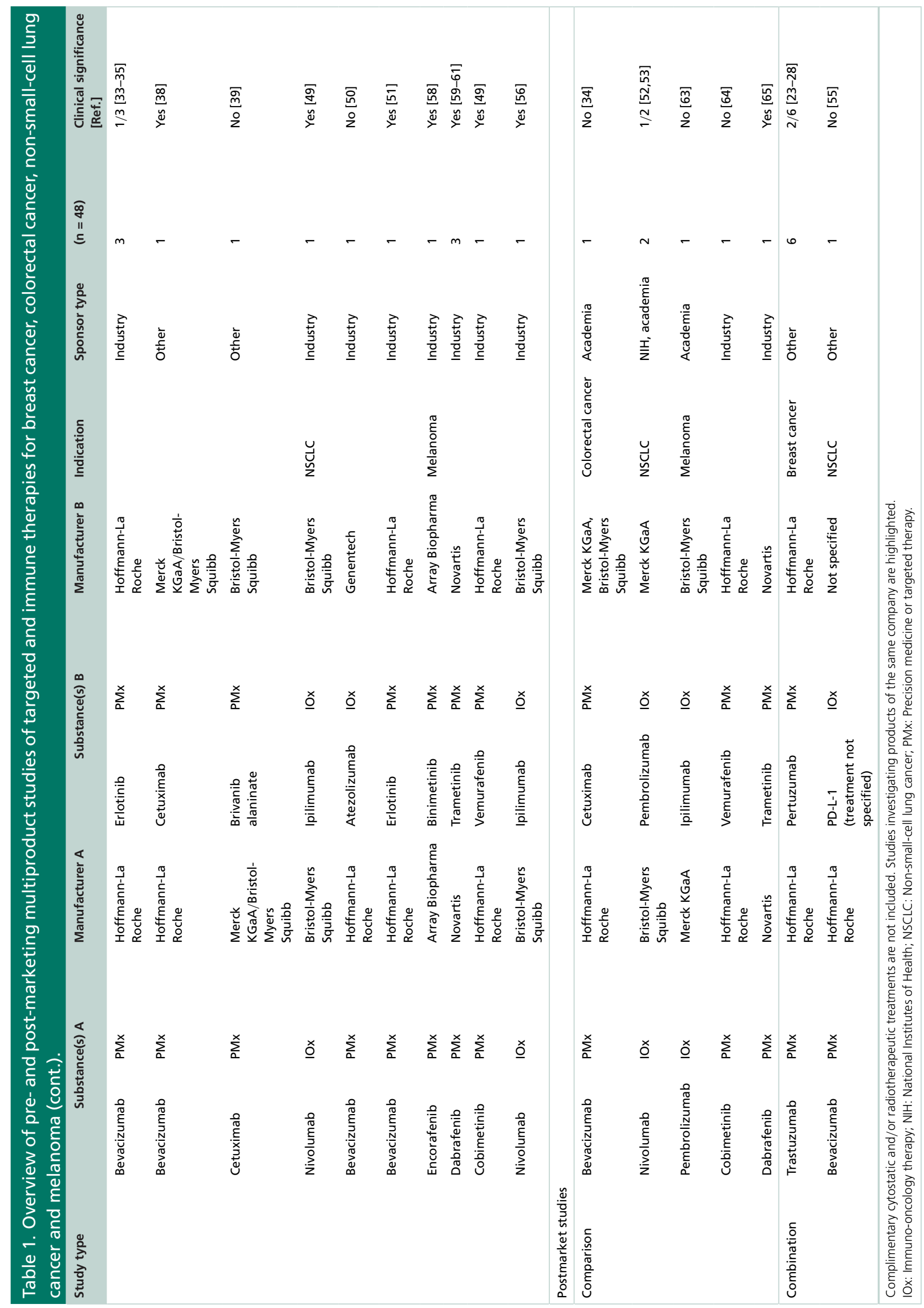




\section{Premarket studies}

Two industry-funded Phase III comparative studies were found that investigated efficacy differences between trastuzumab and lapatinib (a tyrosine kinase inhibitor that inhibits the activity of HER1 and HER2) [14,15]. One compared trastuzumab-emtansine, an antibody-drug conjugate consisting of trastuzumab linked to the cytotoxic agent emtansine, with lapatinib [16,17]. Furthermore, six Phase III combination studies were found. Two of these assessed the benefits of treatment with trastuzumab plus pertuzumab (a HER2 monoclonal antibody) [6,18], while the other four focused on trastuzumab plus lapatinib [19], trastuzumab plus bevacizumab (a recombinant humanized monoclonal antibody to VEGF) [20], palbociclib (a CDK4/6 kinase inhibitor) plus fulvestrant (an anti-estrogen) [21] and finally abemaciclib (a CDK4/6 kinase inhibitor) plus fulvestrant [22]. All of these studies were industry-sponsored.

\section{Postmarket studies}

In total, six identified postmarket (or real-world) studies were on the combination of innovative medicines in breast cancer. The results of the above landmark clinical trials of the pertuzumab-trastuzumab combination treatment for HER2 metastatic breast cancer patients were found to be similar to those of observational studies performed by the IRCCS Regina Elena National Cancer Institute, the University of Naples, the University of Messina and Genentech [23-26]. Additionally, a retrospective observational study by the Cleveland Clinic Taussig Cancer Institute (2016) and a study funded by Agendia (2017) assessed the combination of pertuzumab with trastuzumab [27,28].

\section{Colorectal cancer}

The mainstay of metastatic colorectal cancer (mCRC) treatment remains the use of fluorouracil and leucovorin combined with irinotecan (FOLFIRI) or oxaliplatin (FOLFOX). The addition of targeted therapies to this standard of care has markedly improved the survival of metastatic colorectal cancer patients [29]. Listed below are the preand postmarketing comparative and/or combination studies on innovative medicines for colorectal cancer.

\section{Premarket studies}

First, three Phase III trials were found to perform comparative investigations of mCRC. Two of these performed head-to-head comparisons of bevacizumab and cetuximab (an EGFR inhibitor) were sponsored by the industry [30] and the Alliance of Clinical Trials in Oncology [31]. The other comparative study was a head-to-head comparison of panitumumab (an EGFR inhibitor) and cetuximab funded by the industry [32].

Second, seven combination studies were identified for CRC. Three of these investigated the benefits of combined maintenance therapy with bevacizumab and erlotinib (an EGFR inhibitor) and were industry sponsored [33-35]. The other Phase III studies investigated bevacizumab plus panitumumab [36], the triplet combination of encorafinib (a BRAF inhibitor) plus cetuximab plus binimetinib (a MEK inhibitor) [37], bevacizumab plus cetuximab [38] and cetuximab plus brivanib alaninate (an inhibitor of both VEGF receptor and FGF receptor) [39] and were either industry-funded or sponsored by other initiatives.

\section{Postmarket studies}

A registry-based observational cohort study from the Sun Yat-sen University Cancer Center (2016) investigated the comparative effectiveness and safety of bevacizumab and cetuximab in mCRC patients from China. The results confirmed the similar effectiveness of the two treatment regimens, as earlier reported by the Phase III Fire-3 study, but showed an increased benefit for patients with peritoneal dissemination using bevacizumab [40].

\section{Non-small-cell lung cancer}

Recent therapeutic advances have also allowed the targeting of molecules in NSCLC treatment [41]. Furthermore, the advent of immunotherapy with anti-PD1 therapies [42], such as pembrolizumab, nivolumab, atezolizumab and anti-CTLA4 therapy ipilimumab [43], has altered the landscape of the management of NSCLC, offering the possibility of long-lasting responses.

\section{Premarket studies}

We found a direct comparison examining osimertinib (an EGFR inhibitor) versus bevacizumab [44] and four industry-funded direct comparisons assessing brigatinib (an ALK and EGFR inhibitor) versus crizotinib (an ALK tyrosine kinase inhibitor) [45], alectinib (an ALK tyrosine kinase inhibitor) versus crizotinib [46], afatinib (an 
EGFR inhibitor) versus erlotinib [47] and icotinib (an EGFR tyrosine kinase inhibitor) versus gefitinib (an EGFR inhibitor) [48]. Second, three industry-funded combination studies were discovered, investigating nivolumab plus ipilimumab [49], bevacizumab plus atezolizumab (an anti-PD-L1 antibody) [50] and bevacizumab plus erlotinib [51].

\section{Postmarket studies}

Two postmarketing comparative studies at the Irish Regional Cancer Center (2018) [52] and the University Hospital of Zürich (2018) [53] assessed the differences between nivolumab and pembrolizumab in real-world NSCLC patients. Their findings revealed that both immune therapies had similar effectiveness. Also, a retrospective real-life study at the General Hospital of Beijing analyzed the efficacy and safety of PD-1 inhibitors (treatment not specified) in combination with bevacizumab [54].

\section{Melanoma}

The incidence rate of melanoma is increasing rapidly [12], causing the vast majority of skin cancer deaths and mainly affecting young adults [55], leading to huge investments in innovative treatments. Currently, anti-PD1 therapy with nivolumab or pembrolizumab is the standard treatment in patients with BRAF wild-type advanced melanoma [56,57]. The anti-CTLA-4 therapy ipilimumab is also an option in patients with metastatic melanoma [55]. Additionally, BRAF inhibitors, such as dabrafenib, vemurafenib and encorafenib, and MEK inhibitors, for instance binimetinib, trametinib and cobimetinib, have significantly expanded melanoma treatment options [58,59].

\section{Premarket studies}

Two direct comparisons (industry-funded) were identified for melanoma. The first investigated the differences between pembrolizumab and ipilimumab [57] and the second considered vemurafenib (a BRAF inhibitor) as compared with the encorafenib and binimetinib combination (a BRAF and an MEK kinase inhibitor, respectively) [58]. Our results also isolated six industry-funded Phase III combination studies. The first examined the combination of encorafenib plus binimetinib [57], three studied dabrafenib plus trametinib (a BRAF and an MEK inhibitor, respectively) [59-61], one investigated the combination of cobimetinib (a MEK inhibitor) plus vemurafenib [62] and, finally, one evaluated nivolumab plus ipilimumab [56].

\section{Postmarket studies}

Three postmarketing combination studies were found to address metastatic melanoma patients: one real-world study of the combination therapy of pembrolizumab plus low-dose ipilimumab [63]; one assessment of cobimetinib in combination with vemurafenib [64]; and last one real-world study evaluating the efficacy of combined dabrafenib and trametinib [65].

\section{Discussion}

In total, 48 studies were found that assessed comparative and/or combined effects of targeted or immune therapies in breast cancer, colorectal cancer, NSCLC and melanoma. To evaluate efficacy and/or effectiveness, such comparative studies should conduct head-to-head comparisons of the novel product with equivalent products, which may be the current standard treatment [66]. Likewise, combination studies should compare the concomitant treatment with the single treatment with one of these products [66]. These combinations should be based on specific rationales supported by research data and should not be restricted by competition, that is, by enabling comparative studies across drug manufacturers. Ultimately, when their outcomes show further therapeutic benefits, for example when the resultant OS, PFS, QoL and cost-effectiveness data are favorable, such investigations may greatly assist regulatory and reimbursement processes [67].

The first published comparative analysis (2012) assessed the differences between trastuzumab-emtansine and lapatinib through a Phase III trial in breast cancer patients [16]. Since then, 12 more comparative RCT studies have followed. Presumably, these were imitated when additional evidence was required for marketing or subsequent reimbursement approval. As a consequence, these studies were found to be industry funded, that is, by the manufacturer applying for marketing authorization. Thus, as the responsibility for conducting such multiproduct assessments lies in the hands of the manufacturer, high RCT costs and intense market competition can prevent such comparative research, despite the promising insights and benefits it can provide for healthcare decision-making. This is especially true for marketed and reimbursed medicinal products. 
Likewise, the first Phase III study evaluating the combined effect of innovative products was published in 2009 (the bevacizumab plus panitumumab Phase III RCT for colorectal cancer) [36]. Since then, 21 more combinatory RCTs have been carried out on the specified cancer indications and many more, including concomitant treatments consisting of more than three novel products, could be investigated. Again, most of these studies were industry funded. However, the majority of the combination RCTs (16 out of 22) involved medicinal products from the same manufacturer. Hence, such assessments are predominantly performed for in-house medicines as they can broaden their targeted patient populations in existing indications and/or provide new opportunities to new cancer indications (also referred to as drug repurposing) by use 'in combination with' as opposed to 'as an alternative to'. Consequently, this finding may imply that the most promising concomitant treatments are not always being tested. Among other reasons, this is caused by the intense market competition for innovative therapies in oncology.

Last, only 13 postmarketing studies were found, of which six specifically investigated the trastuzumab plus pertuzumab combination. Hence, we can acknowledge that much more work could be done on comparative and combined treatment effectiveness. Since it is difficult to test all comparisons of novel (competitive) products during RCTs, we believe that multiproduct assessments in real-life clinical practice are absolutely necessary. This is especially so as real-world data (RWD), when registered, are often the only existing source of evidence on long-term costeffectiveness [68], essential for innovative therapies and required by regulatory agencies [69]. Additionally, regulatory approval of new indications and new concomitant therapies for marketed products can be supported by RWD in situations in which the (combined) treatments' efficacy has already been demonstrated by off-label usage [70]. The need for real-world assessments is reinforced by the emergence of outcome-based managed entry agreements, which provide patients with early access to the most effective but highly expensive (concomitant) treatments by balancing affordability to therapeutic value [69]. Accordingly, we believe that such RWD-based projects could greatly facilitate multiproduct effectiveness and cost-effectiveness assessments of (early) marketed targeted and immune therapies, within and across different manufactures, and should therefore be encouraged.

Furthermore, there is an urgent need to collect evidence on the best treatment strategies in translational research and to invest in the development of models that can help in predicting therapy response. Such translational data could allow investment in the most meaningful studies and pave the way for sequential therapies by early treatment intervention, thereby optimizing patient care and reducing exorbitant treatment costs. Therefore, the collection of samples during pre- and post-market studies for translational research by an independent research institution should be encouraged in order to obtain maximum information from clinical trials. Hence, performing such RWDbased studies could initiate new RCTs if necessary and ultimately guide clinical treatment guidelines to optimize individual patient treatment pathways.

However, our study does not come without limitations. Specifically, this review focused on published studies only (a nonexhaustive overview of ongoing Phase III/IV studies is included in the appendix). In light of the increasing availability and emerging pipeline of targeted and immune therapies in oncology, a future update of this literature review would be well advised. On top of that, this review focused on direct comparison studies only. Indirect comparisons or meta-analyses may also have potential value to inform healthcare decision-making, although currently they are not routinely accepted by regulatory agencies except when they demonstrate noninferiority [71].

\section{Conclusion}

While comparative studies on (concomitant) innovative cancer therapies have been studied far too little across different manufacturers, they are extremely important for healthcare decision-makers to manage therapeutic innovation at an acceptable cost $[8,67]$. In this scenario, RWD are starting to be recognized as a powerful repository of evidence, because regulatory agencies might utilize evidence derived from real-world outcomes to support regulatory decisions [69,70], and because practitioners might employ such evidence on therapeutic cost-effectiveness in single and concomitant therapies to optimize treatment pathways. Furthermore, such assessments could be a way to gather translational data for use in predicting therapy response, that is, to identify mechanisms of (non)response and new therapeutic targets of action. This can initiate new small proof of concept studies, which could fasten innovative treatment development, and guide therapeutic treatment practices.

Unfortunately, the absence of an appropriate regulatory framework enforcing postmarketing comparative studies and concomitant therapy pilot projects, together with related high costs and intensive market competition, currently prevents cross-border multiproduct assessment studies. Therefore, we encourage the initiation and conduct of realworld studies by trusted third parties. To enable this, oncologists should have access to appropriate data through disease registries and should have incentives to conduct translational research using RWD. 


\section{Future perspective}

With the growing importance of real-world evidence and translational research on medicinal products [66-70] together with the development of good procedural practices for observational and/or comparative studies by the International Society for Pharmacoeconomics and Outcomes Research (ISPOR) and International Society for Pharmacoepidemiology (ISPE) special taskforce [68], we expect that future comparative assessments will include more real-world research and will follow these procedural practice guidelines to increase study credibility. Moreover, we expect more involvement of independent research institutions during these future RWD studies.

\section{Executive summary}

\section{Background}

- Emerging cancer knowledge has initiated an era of highly expensive and intensely competing medicines in oncology. Information on their comparative (concomitant) effects is therefore extremely valuable.

- A literature review of relevant clinical studies on targeted and immune therapies was conducted in four cancer domains (breast cancer, colorectal cancer, lung cancer and melanoma) to grasp the importance of this phenomenon and to summarize the types of study sponsor.

\section{Results}

- Systematic searches of PubMed, EMBASE, the Cochrane Central Register of Controlled Trials library and gray literature for May to September 2018 yielded a total of 1455 results; 48 comparative and/or combined treatment studies were included in this review.

- Grouping of these publications resulted in 35 comparison or combination therapy groups (28 premarketing and seven postmarketing studies).

Conclusion

- Comparative effects are mainly assessed in clinical studies when required by regulators.

- Combination effects are mainly assessed in clinical studies on products of the same market authorization holder.

- Real-world studies can facilitate the generation of such evidence and could be used to seek new (translational) information to initiate additional RCTs and guide treatment decision pathways.

\section{Supplementary data}

To view the supplementary data that accompany this paper please visit the journal website at: www.futuremedicine.com/doi/full/ 10.2217/cer-2018-0131

\section{Acknowledgments}

This literature review benefitted from the review of $L$ Brochez, dermato-oncologist in the Department of Dermatology, Ghent University Hospital, Belgium and steering member of the Immuno-Oncology Network Ghent (ION) and the Cancer Research Institute Ghent (CRIG).

Financial \& competing interests disclosure

Financial support for this study was provided entirely by a grant from the Vlerick Business School. The funding agreement ensured the authors' independence in designing the study, interpreting its results and publishing the report. The authors have no other relevant affiliations or financial involvement with any organization or entity with a financial interest in or financial conflict with the subject matter or materials discussed in the manuscript apart from those disclosed.

No writing assistance was utilized in the production of this manuscript.

\section{References}

Papers of special note have been highlighted as: $\bullet$ of interest; $\bullet \bullet$ of considerable interest

1. De Bono JS, Ashworth A. Translating cancer research into targeted therapeutics. Nature 467(7315), 543 (2010).

- Paper regarding a new era of innovative medicines in oncology: targeted therapy.

2. Khalil DN, Smith EL, Brentjens RJ, Wolchok JD. The future of cancer treatment: immunomodulation, CARs and combination immunotherapy. Nat. Rev. Clin. Oncol. 13, 273-290 (2016).

- Paper regarding a new era of innovative medicines in oncology: immune therapy.

3. Aronson JK, Ferner RE, Hughes DA. Defining rewardable innovation in drug therapy. Nat. Rev. Drug Disc. 11(4), 253-254 (2012).

4. DeVita VT Jr, Young RC, Canellos GP. Combination versus single agent chemotherapy: a review of the basis for selection of drug treatment of cancer. Cancer 35(1), 98-110 (1975). 
5. Chabner BA, Roberts TG Jr. Timeline: chemotherapy and the war on cancer. Nat. Rev. Cancer 5(1), 65-72 (2005).

6. Swain SM, Baselga J, Kim SB et al. Pertuzumab, trastuzumab, and docetaxel in HER2-positive metastatic breast cancer. N. Engl. J. Med. 372(8), 724-734 (2015).

- Well-known example of a study on improved therapeutic benefits when combining targeted therapies.

7. Durkee BY, Qian Y, Pollom EL et al. Cost-effectiveness of pertuzumab in human epidermal growth factor receptor 2-positive metastatic breast cancer. J. Clin. Oncol. 34(9), 902-909 (2016).

8. Dakin H, Gray A. Decision-making for healthcare resource allocation: joint v. separate decisions on interacting interventions. Med. Decis. Mak. 38(4), 476-486 (2018).

-• Demonstrates the importance of making separate decisions for combinatory treatments.

9. Kelly RJ, Smith TJ. Delivering maximum clinical benefit at an affordable price: engaging stakeholders in cancer care. Lancet Oncol. 15, e112-e118 (2014).

10. Hill A, Redd C, Gotham D et al. Estimated generic prices of cancer medicines deemed cost-ineffective in England: a cost estimation analysis. BMJ Open 7, e011965 (2017).

11. DiMasi JA, Hansen RW, Grabowski HG. The price of innovation: new estimates of drug development costs. J. Health Econ. 22(2), 151-185 (2003).

- Study on the high costs of innovative medicinal products.

12. Sleijfer S, Verweij J. Health policy: affordability of drugs used in oncology health care. Nat. Rev. Clin. Oncol. 13(6), 331-332 (2016).

13. Siegel RL, Miller KD, Jemal A. Cancer statistics, 2018. CA Cancer J. Clin. 68(1), 7-30 (2018).

14. Gelmon KA, Boyle FM, Kaufman B et al. Lapatinib or trastuzumab plus taxane therapy for human epidermal growth factor receptor 2-positive advanced breast cancer: final results of NCIC CTG MA.31. J. Clin. Oncol. 33(14), 1574-1583 (2015).

15. Piccart-Gebhart M, Holmes E, Baselga J et al. Adjuvant lapatinib and trastuzumab for early human epidermal growth factor receptor 2-positive breast cancer: results from the randomized Phase III adjuvant lapatinib and/or trastuzumab treatment optimization trial. $J$. Clin. Oncol. 34(10), 1034-1042 (2016).

16. Verma S, Miles D, Gianni L et al. Trastuzumab emtansine for HER2-positive advanced breast cancer. N. Engl. J. Med. 367(19), 1783-1791 (2012).

17. Dieras V, Miles D, Verma $S$ et al. Trastuzumab emtansine versus capecitabine plus lapatinib in patients with previously treated HER2-positive advanced breast cancer (EMILIA): a descriptive analysis of final overall survival results from a randomised, open-label, Phase 3 trial. Lancet Oncol. 18(6), 732-742 (2017).

18. Von Minckwitz G, Procter M, de Azambuja E et al. Adjuvant pertuzumab and trastuzumab in early HER2-positive breast cancer. $N$. Engl. J. Med. 377(2), 122-131 (2017).

19. Blackwell KL, Burstein HJ, Storniolo AM. Overall survival benefit with lapatinib in combination with trastuzumab for patients with HER2-positive metastatic breast cancer: final results from the EGF104900 study. J. Clin. Oncol. 30(21), 2585-2592 (2012).

20. Gianni L, Romieu GH, Lichinitser M et al. AVEREL: a randomized phase III trial evaluating bevacizumab in combination with docetaxel and trastuzumab as first-line therapy for HER2-positive locally recurrent/metastatic breast cancer. J. Clin. Oncol. 31(14), 1719-1725 (2013).

21. Iwata H, Im S-A, Masuda N et al. PALOMA-3: Phase III trial of fulvestrant with or without palbociclib in premenopausal and postmenopausal women with hormone receptor-positive, human epidermal growth factor receptor 2-negative metastatic breast cancer that progressed on prior endocrine therapy—safety and efficacy in Asian patients. J. Global Oncol. 3(4), 289-303 (2017).

22. Sledge GW, Toi M, Neven P et al. MONARCH 2: abemaciclib in combination with fulvestrant in women with HR+/HER2advanced breast cancer who had progressed while receiving endocrine therapy. J. Clin. Oncol. 35(25), 2875-2884 (2017).

23. Gamucci T, Mentuccia L, Sperduti I et al. Efficacy of pertuzumab in combination with trastuzumab and a taxane in first-line treatment for metastatic breast cancer (MBC): a multicenter, retrospective, observational study. J. Clin. Oncol. 35(15 Suppl), e12504 (2017).

24. De Placido S, Giuliano M, Schettini F et al. Human epidermal growth factor receptor 2 dual blockade with trastuzumab and pertuzumab in real life: Italian clinical practice versus the CLEOPATRA trial results. Breast 38, 86-91 (2018).

25. Robert NJ, Goertz H-P, Chopra P et al. HER2-positive metastatic breast cancer patients receiving pertuzumab in a community oncology practice setting: treatment patterns and outcomes. Drugs Real World Outcomes 4(1), 1-7 (2017).

26. Ricciardi G, Ficorella C, Iezzi et al. Efficacy and safety of the combination of pertuzumab (P) plus trastuzumab (T) plus docetaxel (D) for HER-2 positive metastatic breast cancer (MBC) in pretreated patients (pts) with trastuzumab in the neo/adjuvant setting: a real-life study. J. Clin. Oncol. 35 (15 Suppl.), e12516 (2017).

27. Tiwari SR, Mishra P, Raska P et al. Retrospective study of the efficacy and safety of neoadjuvant docetaxel, carboplatin, trastuzumab/pertuzumab (TCH-P) in nonmetastatic HER2-positive breast cancer. Breast Cancer Res. Treat. 158(1), 189-193 (2016).

28. Beitsch P, Whitworth P, Baron P et al. Pertuzumab/trastuzumab/CT versus trastuzumab/CT therapy for HER2+ breast cancer: results from the prospective neoadjuvant breast registry symphony trial (NBRST). Ann. Surg. Oncol. 24(9), 2539-2546 (2017). 
29. Van Cutsem E, Cervantes A, Nordlinger B, Arnold D. Metastatic colorectal cancer: ESMO Clinical Practice Guidelines for diagnosis, treatment and follow-up. Ann. Oncol. 25(Suppl. 3, iii), 1-9 (2014).

30. Heinemann V, von Weikersthal LF, Decker T et al. FOLFIRI plus cetuximab versus FOLFIRI plus bevacizumab as first-line treatment for patients with metastatic colorectal cancer (FIRE-3): a randomised, open-label, Phase 3 trial. Lancet Oncol. 15(10), 1065-1075 (2014).

31. Venook AP, Niedzwiecki D, Lenz $\mathrm{H}$ et al. Effect of first-line chemotherapy combined with cetuximab or bevacizumab on overall survival in patients with KRAS wild-type advanced or metastatic colorectal cancer: a randomized clinical trial. JAMA 317(23), 2392-2401 (2017).

32. Price T, Kim TW, Li J et al. Final results and outcomes by prior bevacizumab exposure, skin toxicity, and hypomagnesaemia from ASPECCT: randomized Phase 3 non-inferiority study of panitumumab versus cetuximab in chemorefractory wild-type KRAS exon 2 metastatic colorectal cancer. Eur. J. Cancer 68, 51-59 (2016).

33. Hagman H, Frodin JE, Berglund A et al. A randomized study of KRAS-guided maintenance therapy with bevacizumab, erlotinib or metronomic capecitabine after first-line induction treatment of metastatic colorectal cancer: the Nordic ACT2 trial. Ann. Oncol. 27(1), $140-147$ (2016).

34. Johnsson A, Hagman H, Frodin JE et al. A randomized Phase III trial on maintenance treatment with bevacizumab alone or in combination with erlotinib after chemotherapy and bevacizumab in metastatic colorectal cancer: the Nordic ACT Trial. Ann. Oncol. 24(9), 2335-2341 (2013).

35. Tournigand C, Chibaudel B, Samson B et al. Bevacizumab with or without erlotinib as maintenance therapy in patients with metastatic colorectal cancer (GERCOR DREAM; OPTIMOX3): a randomised, open-label, phase 3 trial. Lancet Oncol. 16(15), 1493-1505 (2015).

36. Hecht JR, Mitchell E, Chidiac T et al. A randomized Phase IIIB trial of chemotherapy, bevacizumab, and panitumumab compared with chemotherapy and bevacizumab alone for metastatic colorectal cancer. J. Clin. Oncol. 27(5), 672-680 (2009).

37. Van Cutsem E, Cuyle P-J, Huijberts $S$ et al. BEACON CRC study safety lead-in (SLI) in patients with BRAFV600E metastatic colorectal cancer (mCRC): efficacy and tumor markers. J. Clin. Oncol. 36(4 Suppl.), 627 (2018).

38. Tol J, Koopman M, Cats A et al. Chemotherapy, bevacizumab, and cetuximab in metastatic colorectal cancer. N. Engl. J. Med. 360(6), 563-572 (2010).

39. Siu LL, Shapiro JD, Jonker DJ et al. Phase III randomized, placebo-controlled study of cetuximab plus brivanib alaninate versus cetuximab plus placebo in patients with metastatic, chemotherapy-refractory, wild-type K-RAS colorectal carcinoma: the NCIC clinical trials group and AGITG CO.20 trial. J. Clin. Oncol. 31(19), 2477-2484 (2013).

40. Bai L, Wang F, Li Z et al. Chemotherapy plus bevacizumab versus chemotherapy plus cetuximab as first-line treatment for patients with metastatic colorectal cancer: results of a registry-based cohort analysis. Medicine 95(51), e4531 (2016).

41. Gettinger S (Ed.). Targeted therapy in advanced non-small-cell lung cancer. Semin. Respir. Crit. Care Med. 29(3), 291-301 (2008).

42. Topalian SL, Hodi FS, Brahmer JR et al. Safety, activity, and immune correlates of anti-PD-1 antibody in cancer. N. Engl. J. Med. 366(26), 2443-2454 (2012).

43. Lynch TJ, Bondarenko I, Luft A et al. Ipilimumab in combination with paclitaxel and carboplatin as first-line treatment in stage IIIB/IV non-small-cell lung cancer: results from a randomized, double-blind, multicenter phase II study. J. Clin. Oncol. 30(17), 2046-2054 (2012).

44. Nie K, Zhongfa Z, Chunling Z et al. Osimertinib compared docetaxel-bevacizumab as third-line treatment in EGFR T790M mutated non-small-cell lung cancer. Lung Cancer 121, 5-11 (2018).

45. Camidge R, Kim HR, Ahn MJ et al. Brigatinib versus crizotinib in ALK-positive non-small-cell lung cancer. N. Engl. J. Med. doi: 10.1056/NEJMoa1810171 (2018) (Epub ahead of print).

46. Peters S, Cambridge RD, Shaw AT et al. Alectinib versus crizotinib in untreated ALK-positive non-small-cell lung cancer. N. Engl. J. Med. 377, 829-838 (2017).

47. Soria JC, Felip E, Cobo $\mathrm{M}$ et al. Afatinib versus erlotinib as second-line treatment of patients with advanced squamous cell carcinoma of the lung (LUX-Lung 8): an open-label randomised controlled phase 3 trial. Lancet Oncol. 16(8), 897-907 (2015).

48. Yuankai S, Zhang L, Xiaoqing L et al. Icotinib versus gefitinib in previously treated advanced non-small-cell lung cancer (ICOGEN): a randomised, double-blind phase 3 non-inferiority trial. Lancet Oncol. 14(10), 953-961 (2013).

49. Hellmann MD, Ciuleanu TE, Pluzanski A et al. Nivolumab plus ipilimumab in lung cancer with a high tumor mutational burden. $N$. Engl. J. Med. 378(22), 2093-2104 (2018).

50. Socinski MA, Jotte RM, Cappuzzo F et al. Atezolizumab for first-line treatment of metastatic nonsquamous NSCLC. N. Engl. J. Med. 378(24), 2288-2301 (2018).

51. Herbst RS, Ansari R, Bustin F et al. Efficacy of bevacizumab plus erlotinib versus erlotinib alone in advanced non-small-cell lung cancer after failure of standard first-line chemotherapy (BeTa): a double-blind, placebo-controlled, Phase 3 trial. Lancet 377(9780), 1846-1854 (2011).

52. Dennehy C, Mullally W, Goggin C et al. Real world experience with pembrolizumab and nivolumab for treatment of non-small cell lung cancer (NSCLC) in an Irish regional cancer center. J. Clin. Oncol. 36(15 Suppl.), e21196 (2018). 
53. Sanoyan DA, Siebenhüner A, Delaloye R, Bankel L, Paulino TDL, Curioni A. 23P Real-life experience with nivolumab and pembrolizumab in patients(pts) with advanced non-small cell lung cancer (NSCLC): efficacy and safety analysis at the University Hospital Zurich. Ann. Oncol. 28(Suppl. 11), xi7 (2017).

54. Zhang F, Huang D, Zhang Y, Wang G, Cai S, Hu Y. The efficacy and safety of PD-1 inhibitors in combination with or without chemotherapy and/or bevacizumab as second line treatment or beyond in non-small cell lung cancer: preliminary data from a real-world setting. J. Clin. Oncol. 36(15 Suppl.), e21129 (2018).

55. Brochez L, Myny K, Bleyen L et al. The melanoma burden in Belgium: premature morbidity and mortality make melanoma a considerable health problem. Melanoma Res. 9(6), 614-618 (1999).

56. Wolchok JD, Chiarion-Sileni V, Gonzalez R et al. Overall survival with combined nivolumab and ipilimumab in advanced melanoma. N. Engl. J. Med. 377(14), 1345-1356 (2017).

57. Robert C, Schachter J, Long GV et al. Pembrolizumab versus ipilimumab in advanced melanoma. N. Engl. J. Med. 372(26), 2521-2532 (2015).

58. Dummer R, Ascierto PA, Gogas HJ et al. Encorafenib plus binimetinib versus vemurafenib or encorafenib in patients with BRAF-mutant melanoma (COLUMBUS): a multicentre, open-label, randomised Phase 3 trial. Lancet Oncol. 19(5), 603-615 (2018).

59. Robert C, Karaszewska B, Schachter J et al. Improved overall survival in melanoma with combined dabrafenib and trametinib. N. Engl. J. Med. 372(1), 30-39 (2015).

60. Long GV, Stroyakovskiy D, Gogas $\mathrm{H}$ et al. Dabrafenib and trametinib versus dabrafenib and placebo for Val600 BRAF-mutant melanoma: a multicentre, double-blind, Phase 3 randomised controlled trial. Lancet 386(9992), 444-451 (2015).

61. Long GV, Hauschil A, Santinami M et al. Adjuvant dabrafenib plus trametinib in stage III BRAF-mutated melanoma. N. Engl. J. Med. 377(19), 1813-1823 (2017).

62. Ascierto PA, McArthur GA, Dréno B et al. coBRIM: a Phase 3, double-blind, placebo-controlled study of vemurafenib versus vemurafenib + cobimetinib in previously untreated BRAF(V600) mutation-positive patients with unresectable locally advanced or metastatic melanoma (NCT01689519). J. Transl. Med. 13(Suppl. 1), O4 (2015).

63. Kirchberger MC, Moreira A, Erdmann M, Schuler G, Heinzerling L. Real world experience in low-dose ipilimumab in combination with PD-1 blockade in advanced melanoma patients. Oncotarget 9(48), 28903-28909 (2018).

64. Ocvirk J, Rebersek M, Boc M, Mesti T. Patients with metastatic melanoma treated with vemurafenib as monotherapy or in combination with cobimetinib. J. Clin. Oncol. 35(15 Suppl.), e21016 (2017).

65. Algarra SM, Soriano V, Fernandez-Morales L et al. Dabrafenib plus trametinib for compassionate use in metastatic melanoma: a STROBE-compliant retrospective observational postauthorization study. Medicine 96(52), e9523 (2017).

66. Schaumberg DA, McDonald L, Shah S, Stokes M, Nordstrom BL, Ramagopalan SV. Evaluation of comparative effectiveness research: a practical tool. J. Comp. Eff. Res. 7(5), 503-515 (2018).

-. Review on comparative effectiveness research to critically identify high-quality studies.

67. Kleijnen S, Lipska I, Leonardo AT et al. Relative effectiveness assessments of oncology medicines for pricing and reimbursement decisions in European countries. Ann. Oncol. 27(9), 1768-1775 (2016).

68. Berger ML, Sox H, Willke RJ et al. Good practices for real-world data studies of treatment and/or comparative effectiveness: recommendations from the joint ISPOR-ISPE Special Task Force on real-world evidence in health care decision making. Pharmacoepidemiol. Drug Saf. 26(9), 1033-1039 (2017).

-• Good procedural practice recommendations for real-world studies.

69. Eichler HG, Baird LG, Barker R et al. From adaptive licensing to adaptive pathways: delivering a flexible life-span approach to bring new drugs to patients. Clin. Pharmacol. Therapeut. 97(3), 234-246 (2015).

- Explains the concept of adaptive pathways and the importance of real-world evidence.

70. Katkade VB, Sanders KN, Zou KH. Real world data: an opportunity to supplement existing evidence for the use of long-established medicines in health care decision making. J. Multidiscip. Healthc. 2(11), 295-304 (2018).

71. Es-Skali IK, Spoort J. Analysis of indirect treatment comparisons in national health technology assessment assessments and requirements for industry submissions. J. Comp. Eff. Res. 7(4), 397-409 (2018). 